\title{
Investigation on the Potential Rise and Currents in Insulated Mast Scheme with Single Tower During Stroke Interception
}

\author{
Udaya Kumar*
}

High Voltage Laboratory, Department of Electrical Engineering, Indian Institute of Science, Bangalore 560012 , India

\begin{abstract}
The insulated mast scheme for the lightning protection system can be found in a few practical designs. Many advantages over conventional protection system are some times envisaged. However, the technical literature on the analysis of such schemes and further quantification of their protection efficacy is rather scarce. As a first step to address this problem, the present work is taken up and the potential rise at the top and ground end currents in insulating mast scheme with single tower is investigated for several tower heights and pertinent values of other parameters. The quantities that are investigated are the potential difference across the insulation and ground end currents for both tower and the ground wires. Quantifications are carried out for the relevant range of stroke current front times. The influence of number of ground wires, their earthing location and to a limited extent, the length of the insulating support have been ascertained. Some relevant discussion on insulation strength is made. These findings are quite novel and aid in quantification of the practical efficacy of the insulated mast scheme. The level of induction to the support tower and possible flashover to the same are not in favour of this scheme.
\end{abstract}

Keywords: Lightning protection, insulated mast scheme, ground wires, surface breakdown strength, induced tower currents.

\section{INTRODUCTION}

The Lightning Protection System (LPS) for critical structures involves isolated protection schemes. It generally comprises of air termination network, down conductor and earth termination system. The cost of the LPS is evidently determined by its spatial extension and in particular, it is sensitively dependent on the height. For a given height, by taking the LPS closer to the system under protection, the magnitude of current in a bypass/shielding failure stroke could be reduced. However, this is restricted by two aspects. Firstly, the potential rise on LPS during stroke interception should not lead to a flashover to the system under protection. Secondly, the level of electromagnetic fields produced in the protected volume should be well within the permissible limits.

In order to deal with these contradicting requirements, some designs of LPS have resorted to the so-called insulated mast design (refer to Fig. 1). The basic philosophy is to convey the current in the intercepted strokes away from the protected volume. This design involves air termination formed by lightning masts and in distributed system, the interlinking shield wires.

The mechanical support to the air termination is provided by towers, with an insulating /mast cylinder at their top, such that air termination would be electrically isolated from the towers. The down conductor system is formed by catenaries or ground wires, which connect the lightning mast to the remote earth termination network. This type of designs is common in many satellite launch pad lightning protection systems.

*Address correspondence to this author at the High Voltage Laboratory, Department of Electrical Engineering, Indian Institute of Science, Bangalore 560 012, India; E-mail: uday@hve.iisc.ernet.in

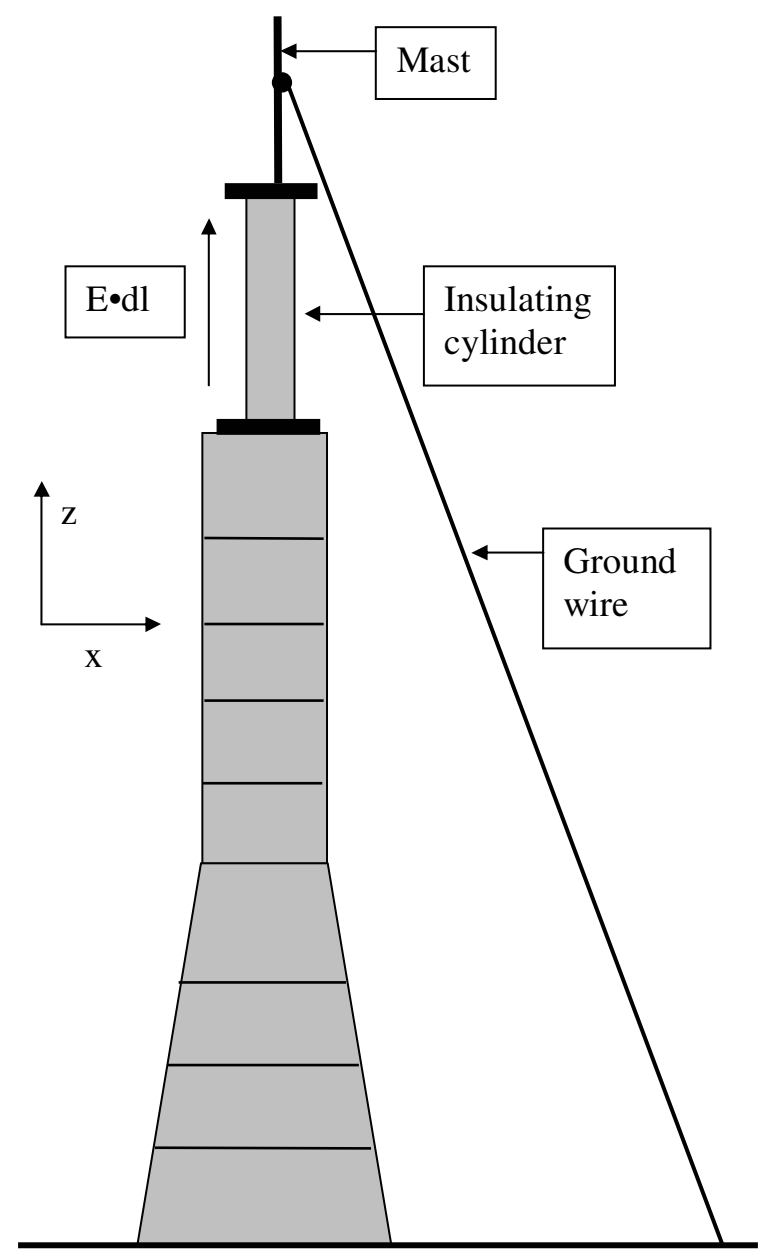

Fig. (1). Schematic of insulated mast design. 
This scheme, if successful, would reduce the following threats. As tower is not the down conductor, rise in its potential and hence the risk of flashover to the system under protection is minimized. Simulation results indicate that the peak values of tower base currents, when the tower is made as a down conductor, are in the range of 100 to $180 \%$ of the injected stroke current [1]. The field measurements on tall towers [2] are supportive of the above range. The induced currents generate significant magnetic fields in the protected volume, as well as, rise in soil potential. On the other hand, if the insulated mast scheme is successful, the resulting electromagnetic fields in the protected volume, the rise in soil potential and gradient at the tower footings are drastically reduced.

However, the success of this scheme is mainly governed by the rise in potential of the LPS and the flashover strength between the support tower and LPS [3]. Inspection of Fig. (1) indicates that there are two distinct air gaps at the top, which need attention. One is obviously across the insulator i.e. between the lightning mast and the support tower and the second one is between the catenary/ground wire and the support tower. The gap length of the latter will generally be in the same range as the former. Due to the presence of these air gaps, the bulk breakdown strength of the insulating support will not be of any use at all. A further discussion on the insulating cylinder will be made later.

The insulated mast scheme seems to have not drawn any serious attention in the technical literature. In fact, the present day knowledge on both the potential rise, as well as, the breakdown strength of the associated gaps for the resulting non-standard impulse voltages is rather limited. Unlike, the air gap seen between the descending stepped leader and the air termination network, the above-mentioned air gaps will be subjected to lightning like fast impulses, however, with oscillatory wave shape. The potential rise for this scheme with a $120 \mathrm{~m}$ tall support tower has been addressed in an earlier work [3]. As only a particular tower height was considered, its results cannot be generalised.

In order to evaluate the possible success of this scheme, a clear knowledge on many aspects is very essential. One of the important aspects among them would be the potential across the two air gaps mentioned earlier. In practice, the possible range of insulator lengths is expected to be within $10 \mathrm{~m}$ and therefore the air gaps discussed earlier must be in the same range. Keeping this in mind, the line integral of electric field across the support insulator can be considered as the potential rise, an index for the dielectric stress. The present work basically deals with the schemes involving single tower. The scope of the work is to evaluate the potential rise across the support insulator for typical range of insulator lengths, height of the support tower and configuration of catenaries/ground wires. Also in the previous work [3], it is shown that the induction to the support tower, especially for strokes with fast fronts, could be significant. In view of the same, the induced tower base currents are also taken as a parameter under study. Even though a limited ground wire configurations is considered, it is deemed to be quite representative of the possible range. Part of this work has been incorporated in the thesis of author's research student [1].

\section{PRESENT WORK}

The primary quantities under study are the potential rise across the top of the supporting tower and the lightning mast, and the induced tower base currents. Obviously not just the peak amplitude, the overall wave shape is also important. In the following first the parameters for the intended study will be briefed.

\subsection{Study Parameters}

The parameters considered for the study are:

\subsubsection{Length of the Insulating Support}

Even though simple logic suggests that the length of the insulator must be as large as possible, several practical consideration limits it to a range of 5-10 m. First of all these insulators cannot be formed in pieces and assembled together [3]. Any metallic joints can aid in flashover. Forming a single piece of large length is limited by the mechanical strength considerations. The load on the insulator is not small. It is subjected to the severe bending stress by the ground wires and to the wind load, mechanical vibration of the system etc. Apart from these, insulator forms a considerable load on the support tower. In view of such considerations typically the length is chosen within the above range. Further, the material for the insulator must be selected considering the UV radiation from Sun, pollution (mostly saline) deposition, aging characteristics etc.

\subsubsection{Height of the Support Tower}

Height of the support tower possesses a direct influence on the rise in potential. It basically governs the time of arrival for the benign negative ("potential or voltage wave") reflection originating from the ground end of the catenaries/ground wires. In order to cover wide range of possible LPS heights, study will be made for the support tower heights of $120 \mathrm{~m}, 90 \mathrm{~m}, 60 \mathrm{~m}$ and $45 \mathrm{~m}$.

\subsubsection{Number of Ground Wires}

In the insulated mast scheme, ground wires/catenaries form the down conductor system. In other words, they are the paths for the discharge current to ground. From the electrical point of view, more number of ground wires seems to be attractive. However, they add on to the mechanical load on the insulation cylinder and hence in turn the load on the tower, as well as, the system cost. In view of this, study is limited to schemes with 1, 2, and 4 ground wires only. Further, they occupy considerable amount of valuable space. The inevitable sag on the ground wires has been neglected.

\subsubsection{Grounding Location of Ground Wires}

The grounding location is constrained by two issues. Firstly, it should be as far as possible so that the field in the protected volume (both at soil and air) would be low. The second requirement is that it should be as close as possible so that the benign negative reflection from the ground end will reach the top in the earliest [3]. It is evident that both are contradictory. Apart from these, there could be local constraints on the grounding location of the ground wires imposed by the presence of other structures/system, cable trenches, roads etc. The locations for ground wire earthing have been chosen such that the net wire length (without sag) 
would be approximately $1.9 H, 1.3 H$, and $1.1 H$ (where $H$ is the height of the tower) and these locations are denoted respectively by $\mathrm{P} 1, \mathrm{P} 2$, and $\mathrm{P} 3$. The actual lengths along with the corresponding vertical angle subtended by the ground wire are given (Table 1). These locations even though quite arbitrary, are selected so as to be in line with an earlier work [3]. In the simulation $6 \mathrm{~mm}$ is taken as the default radius for the ground wire (i.e. $12 \mathrm{~mm}$ diameter).

Table 1. Length of the Ground Wire $L$ in Terms of Height of the Tower $H$ and the Corresponding Angle $\theta$ Made with Respect to the Vertical

\section{(a) Grounding Position P1}

\begin{tabular}{|c|c|c|c|c|c|}
\hline \multicolumn{2}{|c|}{ Height of the Support Tower (m) } & $\mathbf{4 5}$ & $\mathbf{6 0}$ & $\mathbf{9 0}$ & $\mathbf{1 2 0}$ \\
\hline \hline \multirow{2}{*}{ Length of insulating mast $=5 \mathrm{~m}$} & $\mathrm{~L} / \mathrm{H}$ & 1.85 & 1.85 & 1.87 & 1.88 \\
\cline { 2 - 6 } & $\theta^{\circ}$ & 53 & 54 & 55.7 & 56.4 \\
\hline \multirow{2}{*}{ Length of insulating mast $=10 \mathrm{~m}$} & $\mathrm{~L} / \mathrm{H}$ & 1.85 & 1.91 & 1.90 & 1.90 \\
\cline { 2 - 6 } & $\theta^{\circ}$ & 53 & 52.3 & 54.3 & 55.3 \\
\hline
\end{tabular}

(b) Grounding Position P2

\begin{tabular}{|c|c|c|c|c|c|}
\hline \multicolumn{2}{|c|}{ Height of the Support Tower (m) } & 45 & 60 & $\mathbf{9 0}$ & $\mathbf{1 2 0}$ \\
\hline \hline \multirow{2}{*}{ Length of insulating mast $=5 \mathrm{~m}$} & $\mathrm{~L} / \mathrm{H}$ & 1.31 & 1.30 & 1.30 & 1.30 \\
\cline { 2 - 6 } & $\theta^{\circ}$ & 32.1 & 33.8 & 35.9 & 36.8 \\
\hline \multirow{2}{*}{ Length of insulating mast $=10 \mathrm{~m}$} & $\mathrm{~L} / \mathrm{H}$ & 1.31 & 1.37 & 1.35 & 1.34 \\
\cline { 2 - 6 } & $\theta^{\circ}$ & 32.1 & 31.9 & 34.5 & 35.7 \\
\hline
\end{tabular}

(c) Grounding Position P3

\begin{tabular}{|c|c|c|c|c|c|}
\hline \multicolumn{2}{|c|}{ Height of the Support Tower (m) } & $\mathbf{4 5}$ & $\mathbf{6 0}$ & $\mathbf{9 0}$ & $\mathbf{1 2 0}$ \\
\hline \hline \multirow{2}{*}{ Length of insulating mast $=5 \mathrm{~m}$} & $\mathrm{~L} / \mathrm{H}$ & 1.16 & 1.14 & 1.13 & 1.12 \\
\cline { 2 - 6 } & $\theta^{\circ}$ & 16.3 & 17.9 & 20.3 & 21.4 \\
\hline \multirow{2}{*}{ Length of insulating mast $=10 \mathrm{~m}$} & $\mathrm{~L} / \mathrm{H}$ & 1.16 & 1.22 & 1.18 & 1.16 \\
\cline { 2 - 6 } & $\theta^{\circ}$ & 16.3 & 16.7 & 19.4 & 20.6 \\
\hline
\end{tabular}

\subsection{Approach}

For tall towers, its electrical length becomes very much comparable with the wavelengths corresponding to significant higher frequency components of lightning current spectrum. Therefore significant wave propagation effects exist. As the conditions prevailing during return stroke current build up is not conducive to Transverse ElectroMagnetic (TEM) mode, higher order modes like Transverse Magnetic (TM) are expected. However, depending on the height of the system, for the later time periods, TEM and quasi-wave modes can come into existence. In other words, any model to be employed for the analysis should satisfy the governing fields in totality.

In such situations, contribution to the electric field by the time variation in magnetic field becomes quite substantial and therefore the definition and use of scalar electric potential becomes invalid. A detailed analysis of the path dependency of the electric field line integral (potential rise) on down conductor has been made in [4]. However, with regard to the dielectric stress in the gap, the line integral of electric field over the shortest path across the gap would be an appropriate quantifier. Considering its similarity with the definition of classical scalar potential and that the gaps involved are relatively small, it will be termed as the equivalent potential rise or the potential difference (or simply as potential rise). Further, it is worth noting here that the breakdown strength of long air gaps is evaluated only for quasi-static fields in which scalar electric potential is clearly definable.

As the study on natural lightning is impractical, modelling approach is adopted.

\subsubsection{Models Employed in the Literature}

Now the relevant literatures for the methodology that can be employed for the evaluation of required potential rise and currents will be reviewed. Instead of listing out all the literature employing a particular method, only one of them will be quoted. Broadly speaking, the method presented in the literature could be classified into experimental and theoretical.

The experimental approaches involved time domain measurements using either Time Domain Reflectometry technique [5] (which employs a TEM mode approximation to the propagation) or the so-called "Volt-Ampere (V-A)" / "Direct method" [6]. The measurements were made either on the actual towers or on their electromagnetically scaled models. The Volt-Ampere method is more justifiable and is found to give very satisfactory results. Also, studies with scaled models $[5,7]$, while offering acceptable accuracy, are much more economical and less time consuming.

Theoretical approaches employed in the literature are: (i) TEM mode approximation to the wave propagation on simplified geometries [8,9], (ii) Simplified TM mode on simple conductor arrangements [10] and (iii) Use of electromagnetic models (or antenna theory models) [11]. Only the last approach satisfies the governing field equations without resorting to any simplification on the mode of wave propagation or on the overall geometry. Incidentally, due to the electrically thin geometry of the channel, thin wire approximation based on MoM has been widely used in the earlier works for the required numerical field computations [12-14]. In this regard, the public domain code Numerical ElectroMagnetic Code (NEC-2) [15] has been one of the most used tools. This code computes the field in frequency domain and for obtaining time domain quantities, Fourier techniques must be employed [12]. A more detailed description of the approach will be given below.

Invariably, in most of the works, lightning current is assumed to be unaffected by the presence of strike object. This is specifically true for the work pertaining to the lightning surge response of towers and transmission line ground wires. As in most of the standards the unperturbed currents are specified, it is more appropriate to provide the response of the system for the same. In view of these, the channel excitation is modelled by a current source placed at the junction of protection system and the channel. The lightning channel is modelled by a vertical conductor. Only a 
portion of the channel length, which avoids within the observation time period, the arrival of reflection from the remote end, would be adequate for the intended study. This will avoid large computational burden associated with fulllength channel model, as well as, numerical problems associated with the same.

Some difficulties with NEC modelling of the interconnected [16] and complicated tower lattice elements have been reported in earlier works [3]. In view of the same, a previous work employed experimental approach with electromagnetically reduced scale model of $120 \mathrm{~m}$ tall towers. In fact for the problem in hand, the tower top does not get any direct injection, which with short interconnected members has been one of the main source of the problem. Therefore, NEC based approach could be employed reliably for the problem under study. This will not only save time, but also would be very economical.

\subsubsection{Numerical Modelling}

As mentioned earlier, the basic philosophy of modelling is same as that dealt in [12]. For the basic details of the NEC and modelling of the conducting elements one can refer to $[15,17]$. The channel is modelled by a vertical conductor of radius $2.5 \mathrm{~mm}$. A study on simple cylindrical down conductor [1] has shown that the path integral of radial electric field is lower with lower velocities of current propagation. However, in the present configuration where the vertical electric field between the tower top and the ground wire is under consideration, no appreciable dependency on velocity was observed. For the study, by employing resistive-inductive loading, velocity of current propagation along the channel was reduced by a factor of 3 . Similarly, the induced currents in the tower did not exhibit any significant dependency on velocity of current propagation along the channel. In view of these, all the quantification was performed for velocity of propagation equal to the velocity of light (i.e. channel is represented without any loading).

The current source excitation at the centre is realised by a voltage source with a series resistance of $5 \mathrm{k} \Omega$. This value was chosen as most of the literature on tower surge response have employed current source model for the excitation. Also, it facilitates injection of currents specified in the standards. Further, all the frequency domain quantities are normalised with the computed frequency domain source current. As a numerically efficient approach, the channel is brought down to earth at a far distance and to avoid reflection it is terminated with an impedance of $470 \Omega$. It is verified that results are not affected by this modification.

The wavelength $\lambda_{\text {upper }}$ associated with significant higher frequency components of the lightning current spectrum (which is taken as $<=5 \mathrm{MHz}$ ) are much larger than the cross sectional dimensions of the elements of protection system. Therefore, all the conducting elements of the system are considered to be electrically thin and the non-cylindrical elements are replaced with cylindrical elements of equivalent geometric mean radius. All intricacies spanning much less than $\lambda_{\text {upper }}$ are neglected. The ground wire, in order to model the impedance of the earth connection, is terminated with a resistance of $8 \Omega$ and inductance of $5 \mu \mathrm{H}$. This value was considered based on a practical grounding design. For a direct strike, the influence of soil parameters on surge propagation along the protection system seems to be insignificant. Hence, a perfectly conducting earth is considered. The impact of corona is neglected. The experience with transmission lines indicates that they slow down the wave velocity and increase the effective diameter of the conductors. At present, it is not only difficult to include such effects in high frequency codes, but also not necessary, as such a level of sophistication is yet to be reached.

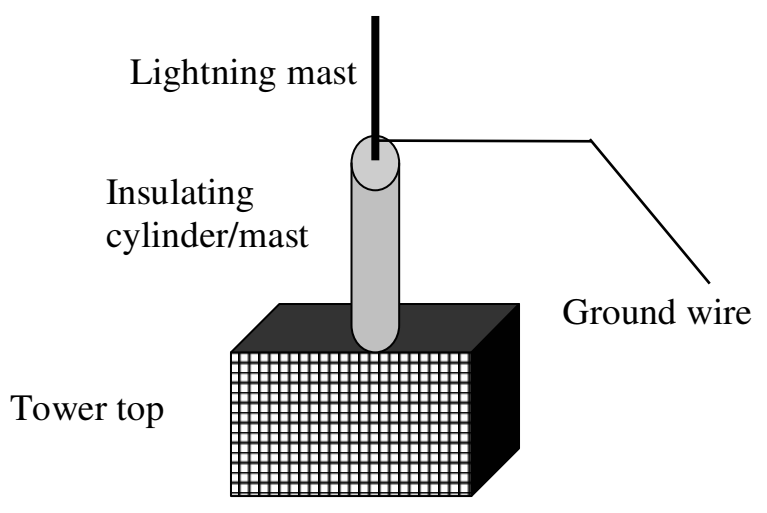

Fig. (2). Schematic of the modified ground wire geometry at the top.

Noting that the tangential component of electric field must be continuous across the dielectric interface and that the insulating cylinder covers very small portion of the conductor, it is assumed that the electric field between the down conductor and tower is not significantly affected. Hence in the modelling, the dielectric cylinder is ignored. This was also essential, as the numerical code employed for the field computation does not handle non-conducting elements. Further, during the evaluation of the field along the horizontal elements of the tower lattice elements in NEC, some numerical errors have been encountered. For this it is suggested that field must be evaluated along the mid of the segments, rather than the ends formed by the junction of several elements [1]. Also to capture the maximum field, evaluation must be made along the radial direction. Keeping these in mind, as shown in figure [2], the catenary/ground wire connection to the mast is slightly modified. Instead of an inclined ground wire trajectory, it is made horizontal at the bottom of the lightning mast and then inclined.

Potential rise across the insulation is evaluated by taking the path integral (in $\mathrm{Z}$ direction) of electric field along the mid of the nearest top horizontal segment of the tower to the above discussed horizontal member added for the modelling. Totally 500 equi-spaced spatial points along z-direction are employed with $1 \mathrm{~cm}$ interval for $5 \mathrm{~m}$ mast case and $2 \mathrm{~cm}$ interval for $10 \mathrm{~m}$ mast case. The NE card in NEC was employed for computing the field. Considering the fine spatial sampling, trapezoidal rule was employed for the numerical integration of the electric field path integral. Later by comparison with the earlier experimental results, it will be shown that this approach is quite adequate.

Totally 1024 frequency samples spanning up to 2.8-5 $\mathrm{MHz}$ has been employed in NEC-2 computation. For saving 
time, many simulations have been run only up to $2.8 \mathrm{MHz}$ and hence results for stroke with a front time of $0.26 \mu \mathrm{s}$ must be considered only as indicative. The source waveform is modeled in a larger window with an initial and final zero padding. These zero padding was very useful in eliminating the offset and low frequency problems, which are encountered in many cases. Also, for frequencies lower than $50 \mathrm{kHz}$, low frequency impedance is directly calculated and incorporated in the calculations. Instead, for these frequencies, if NEC computed values are directly used, then the resulting voltage rise was found to be higher for strokes with front time larger than $7 \mu \mathrm{s}$. However, for strokes with faster front, there was not much change. The time domain quantities are computed in MATLAB using appropriate Fourier techniques. The time step selected for the inversion is in the range of 25-75 ns, with lower value for shorter towers and currents with faster fronts. The overall numerical procedure, for most of the cases, is expected to be within $\pm 5 \%$ of the correct solution. There were occasional problems in the frequency to time domain conversion, which is set right by changing the over all time base and the number of time samples employed for the frequency to time domain inversion.

\section{RESULTS}

Simulations are carried out for potential rise and ground end currents. By neglecting the corona on catenary and soil ionisation at ground, the surge response of LPS can be considered to be linear. In fact, soil ionisation even when present, may not have significant influence for low impedance earth termination. With these, the response of the system can be considered to be independent of stroke current amplitude and polarity. In view of this, for generalisation, all simulation results are presented after normalisation, i.e. peak amplitude of stroke current set to unity.

Two wave shapes were employed in initial testing, double exponential and Gaussian rise - exponential fall. However the maximum rate of rise for both of them was held the same. No significant dependency on the wave shape was observed and hence for all the calculations, latter form is employed. Most of the calculations will be made for strokes with front time (defined based on the rise time between $10 \%$ - $90 \%$ on the rising portion) in the range $0.26 \mu \mathrm{s}, 0.76 \mu \mathrm{s}, 1$ $\mu \mathrm{s}, 2.4 \mu \mathrm{s}, 4.8 \mu \mathrm{s}, 7 \mu \mathrm{s}$ and $17.5 \mu \mathrm{s}$ (Fig. (3) shows the front portion of first 6 currents). These almost encompass the 10$90 \%$ rise time prescribed in IEC [18] for the median rise time of subsequent negative stroke with fast front to first negative stroke with slow fronts.

With regard to the role of tail time of current, simulations results indicate that smaller tail times can increase the potential rise for fast front currents. This increase was within $10-15 \%$. For uniformity across different waveforms, identical tail portion was employed for the simulation. This current tail attained its $50 \%$ after a time period of about 18 $\mu$ s measured from the peak. For the range of tower heights and hence the ground wire lengths considered for the analysis, as the stroke front time is increased, it is found that all the results settle down to their nominal values well within a front time of $7 \mu \mathrm{s}$. In view of the same, results will be presented only up to $4.8 \mu$ s front time. It is also worth mentioning here that due to the low frequency problems with NEC, time domain results will be not so accurate for strokes with very slow front times ( $>7 \mu \mathrm{s})$.

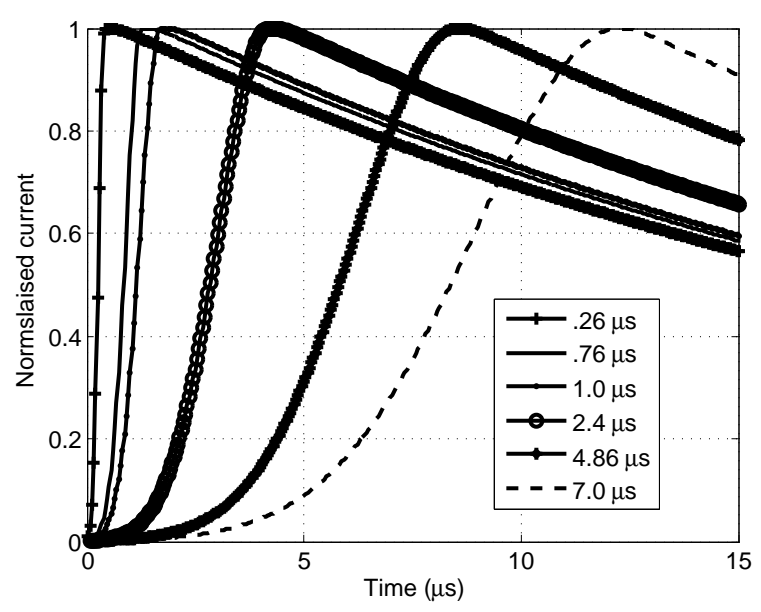

Fig. (3). Front portion of the currents employed in simulation.

Only the results for insulating mast of $5 \mathrm{~m}$ length will be presented in detail. The corresponding values for $10 \mathrm{~m}$ long mast were found to be similar including the temporal characteristics and hence only the potential rise for single ground wire case will be presented.

\subsection{Insulating Mast with One Ground Wire}

Due to its simplicity, this case will be dealt in more detail. More importantly, most of the existing designs, involve only one ground wire and hence it would be more appropriate for a detailed scrutiny.

As mentioned earlier, simulations are carried out for three grounding positions $\mathrm{P} 1, \mathrm{P} 2$ and $\mathrm{P} 3$. The potential rise across the gap, and ground end currents are computed for different front times. Typical time domain results for grounding at P1 are presented in Fig. (4). In the figure, for a better presentation, the top voltage rise and induced tower base currents are plotted with polarity reversed. In Fig. (4a), the rise in voltage has been given for fast $(0.76 \mu \mathrm{s})$ and medium-front $(2.4 \mu \mathrm{s})$ stroke currents. The ground end currents of the ground wire and the tower are given in Fig. $(4 \mathbf{b}, \mathbf{c})$, respectively for stroke with front times 0.76 and 2.4 $\mu \mathrm{s}$.

The current plots clearly indicate the modulation caused by the ground end and channel end reflections on the ground wire. When the two-way travel time along the wire is longer than the time to peak of the stroke current, significant oscillations could be seen, while for the other case it is more like a superimposed oscillations on an impulse wave. The frequency of oscillations in current waveform is given approximately by $(4 / c) \times$ length of the ground wire, where $c$ is the velocity of light in air.

The dynamic electric field across the gap is contributed by retarded charge and time derivative of the retarded current. Therefore, it is dependent on the type of reflections on either end of the ground wire. When the two way travel 
(4a) Potential rise across the insulation for strokes with two different front times.

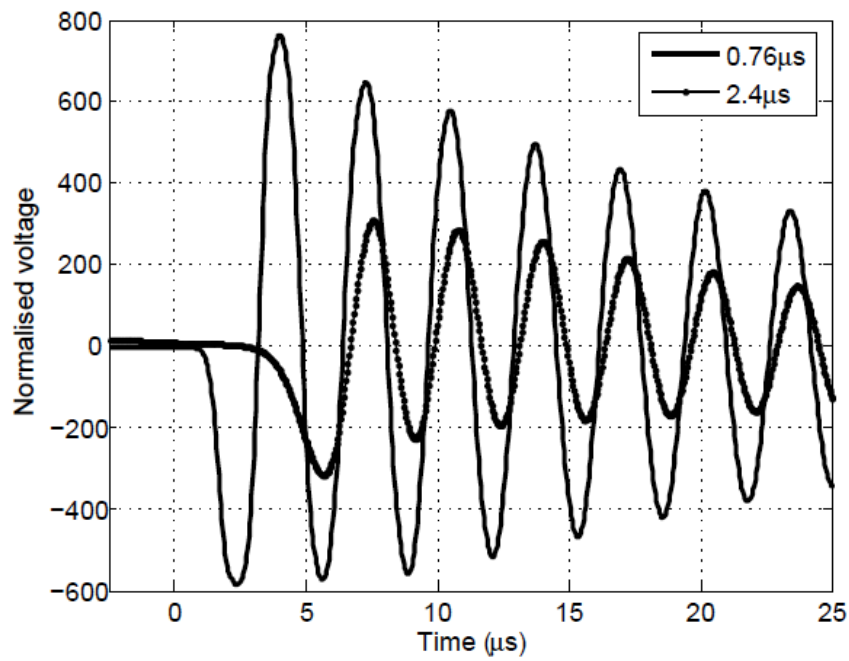

(4b) Ground end currents for stroke with front time of $0.76 \mu \mathrm{s}$

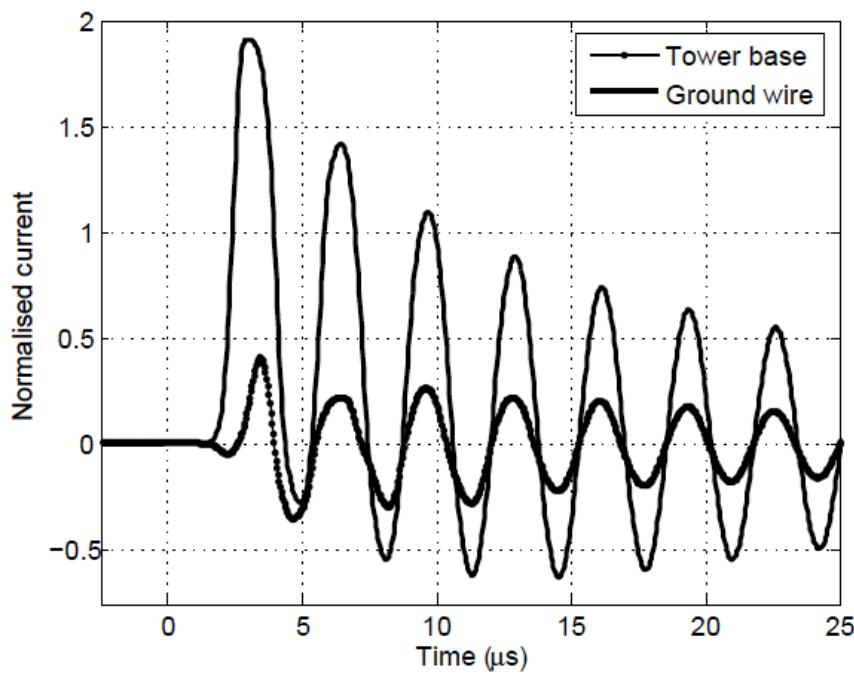

(4c) Ground end currents for stroke with front time of $2.4 \mu \mathrm{s}$.

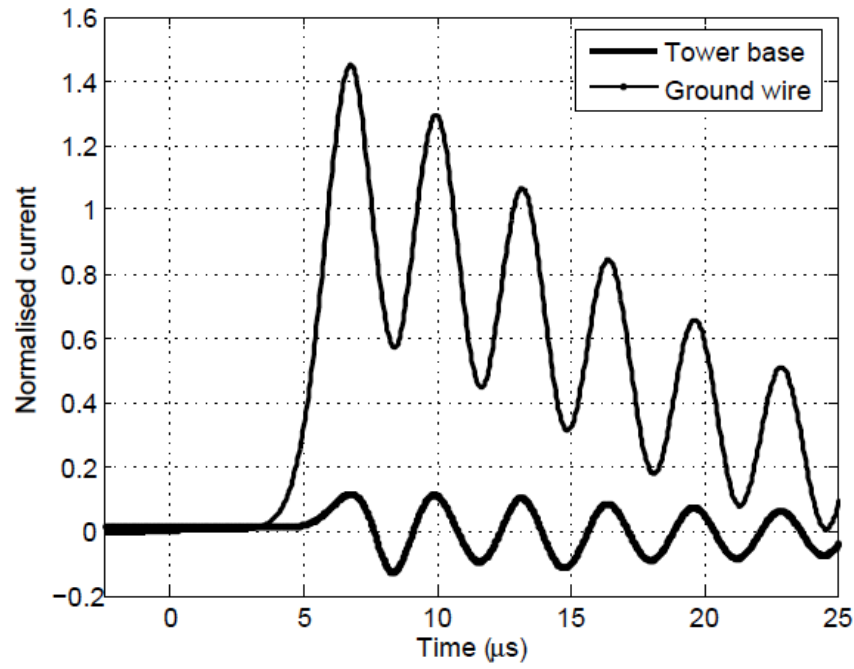

Fig. (4). Results for insulated mast scheme with single ground wire earthed at P1 (Tower height $=120 \mathrm{~m}$, Insulation length $=5 \mathrm{~m}$, Stroke current peak amplitude normalised to unity). time is longer than front time of the stroke current, after the two two-way trips of current wave along the wire, charge builds up and the resulting voltage can exceed even the voltage for the rising portion of the current (refer to Fig. 4a). However, this feature will disappear with increase in front time of the stroke current. For example, voltage rise for the stroke current with $2.4 \mu$ s front time can be seen to have at the beginning almost equal swing on either side.

Table 2. Potential Rise Across Insulation in Volts For Design with one Ground Wire (Stroke Current Peak Amplitude Normalised to Unity \& Insulation Cylinder Length $=5 \mathrm{~m}$ )

(a) Grounding Position P1

\begin{tabular}{|c|c|c|c|c|}
\cline { 1 - 2 } Height of the Support Tower $(\mathbf{m})$ & \multirow{2}{*}{$\mathbf{4 5}$} & $\mathbf{6 0}$ & $\mathbf{9 0}$ & $\mathbf{1 2 0}$ \\
\cline { 1 - 4 } Rise Time $(\boldsymbol{\mu s})$ & & & & \\
\hline \hline 0.26 & 385 & 635 & 705 & 734 \\
\hline 0.75 & 264 & 394 & 576 & 649 \\
\hline 1 & 79 & 138 & 206 & 334 \\
\hline 2.4 & 31 & 70 & 75 & 134 \\
\hline 4.87 & & &
\end{tabular}

(b) Grounding Position P2

\begin{tabular}{|c|c|c|c|c|}
\hline \multirow{yynn}{*}{$\begin{array}{c}\text { Height of the Support Tower }(\mathbf{m}) \\
\text { Rise Time }(\boldsymbol{\mu s})\end{array}$} & \multirow{4}{*}{$\mathbf{6 5}$} & $\mathbf{6 0}$ & $\mathbf{9 0}$ & $\mathbf{1 2 0}$ \\
\hline 0.26 & 549 & 576 & 654 & 694 \\
\hline 0.75 & 247 & 354 & 511 & 588 \\
\hline 1 & 166 & 255 & 418 & 526 \\
\hline 2.4 & 51 & 85 & 120 & 189 \\
\hline 4.87 & 19 & 42 & 45 & 71 \\
\hline
\end{tabular}

(c) Grounding Position P3

\begin{tabular}{|c|c|c|c|c|}
\hline Height of the Support Tower (m) & \multirow{2}{*}{45} & \multirow{2}{*}{60} & \multirow{2}{*}{90} & \multirow{2}{*}{120} \\
\hline Rise Time $(\mu s)$ & & & & \\
\hline 0.26 & 494 & 514 & 610 & 664 \\
\hline 0.75 & 201 & 288 & 442 & 512 \\
\hline 1 & 133 & 201 & 339 & 446 \\
\hline 2.4 & 41 & 66 & 87 & 145 \\
\hline 4.87 & 18 & 32 & 34 & 55 \\
\hline
\end{tabular}

More importantly, as also shown in [3], for fast front stroke currents there exists a considerable amount of induction to the support tower. Internally, the induced currents travel in either direction along the tower and will get negatively reflected at the top and positively at the ground end. However, due to the strong influence of current in the ground wire, the current oscillation along the tower is in synchronism with the former, rather than its own natural 
frequency. This has been verified by considering a hypothetical case in which the ground wire is terminated with resistance in the range of 396-470 $\Omega$. Under such condition, the reflection from the ground end of the wire becomes insignificant and hence oscillations on the ground wire current, as well as, the potential rise will almost disappear. As the accuracy of the computed currents is $\pm 5 \%$ of the input stroke current, induced currents below $10 \%$ must be taken as an indicative value. In other words it must be considered only for ascertaining the level of induction, rather than actual quantifications.

The oscillations in the voltage rise and also the oscillations in the ground end current of the wire are sustained for quite a long time, which may be partly attributed to the consequence of a current source representation for the excitation. In this aspect, they look similar to that on the transmission line. This can be attributed to the relatively low earth termination resistance.

The Table 2 presents the peak amplitude of the potential rise across insulation support of $5 \mathrm{~m}$ length for towers of different heights. A comparison with earlier work (on $120 \mathrm{~m}$ tall tower) [3] would be a good indicator for the adequacy of the modelling. However, in the earlier work, which is based on experimental approach, the potential lead wire method was employed for deriving the potential. On the other hand, in the present work, the potential difference, which is the correct indicator for the stress across the gap, is being evaluated. The values computed are found to be either equal or slightly higher (within 15\%) than the earlier work. Therefore in principle the present modelling is accurate.

The potential rise across the gap can be seen to be dependent on several factors, such as front time of the stroke current, height of the support tower, grounding location of the catenary/ground wire. Even though there are some minor variations (i.e. within $\pm 5 \%$, accuracy of the present approach), which can also be related to time of arrivals and front times, the overall characteristics can be identified as:

- $\quad$ Potential rise increases with decrease in time to crest. If the front time or more correctly, the span of fast rising portion of the stroke current waveform is below the twoway travel time along the catenary/ground wire, potential rise is significant. While in other cases, the benign reflections from the ground end would arrive at the top well before the current peak, thereby reducing the rise in potential. As a result, in this regime, the potential rise exhibits a small dependency on the front time.

- Due to the shorter ground wire length and hence a smaller time for arrival of benign ground end reflection, the rise in potential seems to be least for P3 (the grounding location close to the support tower).

- In general, the potential rise across the gap increases with height of the support tower. With the tower height, the length of the catenary/ground wire also increases and hence the reason quoted above apply equally well to this case.

- The potential rise across the gap, as compared to the potential rise on support tower when used as the down conductor [3], is higher by a factor of 3.0-1.4.
Table 3. Peak Values of Ground End Currents of the Ground Wire in Amperes for Insulated Mast Design with One Ground Wire (Stroke Current Peak Amplitude Normalised to Unity \& Insulation Cylinder Length $=5 \mathrm{~m}$ )

(a) Grounding Position P1

\begin{tabular}{|c|c|c|c|c|}
\hline Support Tower Height (m) & \multirow{2}{*}{45} & \multirow{2}{*}{60} & \multirow{2}{*}{90} & \multirow{2}{*}{120} \\
\hline Rise Time $(\mu s)$ & & & & \\
\hline 0.26 & 1.92 & 1.95 & 1.92 & 1.93 \\
\hline 0.75 & 1.63 & 1.80 & 1.90 & 1.92 \\
\hline 1 & 1.40 & 1.62 & 1.84 & 1.91 \\
\hline 2.4 & 1.04 & 1.08 & 1.24 & 1.45 \\
\hline 4.87 & 1.01 & 1.02 & 1.04 & 1.09 \\
\hline
\end{tabular}

(b) Grounding Position P2

\begin{tabular}{|c|c|c|c|c|}
\cline { 1 - 4 } Support Tower Height $(\mathbf{m})$ & \multirow{2}{*}{$\mathbf{4 5}$} & $\mathbf{6 0}$ & $\mathbf{9 0}$ & $\mathbf{1 2 0}$ \\
\cline { 1 - 4 } Rise Time $(\boldsymbol{\mu} \mathbf{s})$ & 1.96 & 1.90 & 1.92 & 1.94 \\
\hline \hline 0.26 & 1.41 & 1.60 & 1.80 & 1.86 \\
\hline 0.75 & 1.22 & 1.37 & 1.65 & 1.80 \\
\hline 1 & 1.02 & 1.04 & 1.10 & 1.21 \\
\hline 2.4 & 1.01 & 1.01 & 1.02 & 1.04 \\
\hline 4.87 & &
\end{tabular}

(c) Grounding Position P3

\begin{tabular}{|c|c|c|c|c|}
\hline \multirow{yynnn}{*}{ Support Tower Height (m) } & \multirow{2}{*}{$\mathbf{4 5}$} & $\mathbf{6 0}$ & $\mathbf{9 0}$ & $\mathbf{1 2 0}$ \\
\hline \hline 0.26 & & & & \\
\hline 0.75 & 1.91 & 1.89 & 1.96 & 1.96 \\
\hline 1 & 1.33 & 1.50 & 1.74 & 1.84 \\
\hline 2.4 & 1.16 & 1.29 & 1.56 & 1.73 \\
\hline 4.87 & 1.02 & 1.03 & 1.07 & 1.14 \\
\hline & 1.00 & 1.02 & 1.02 & 1.03 \\
\hline
\end{tabular}

The breakdown strengths of the two gaps encountered in the problem are not readily available. The gap between the inclined ground wire and the tower top, which is shown in Fig. (2), is different from the standard gaps associated with high voltage power transmission lines and substations. With regard to the gap across support insulator, unlike the string insulators dealt in high voltage engineering, the interface is mostly along the field line. During rain/mist the whole surface will get wet. The small groove made on the surface for vortex shedding is too small for increasing the net creepage length. Therefore its surface flashover strength whether under clean/wet/polluted conditions cannot be compared with the normal insulator strings and can be expected to be lower. In light of these, the effective breakdown strength of the gap system for positive polarity 
can be expected to be in the range of $500 \mathrm{kV} / \mathrm{m}$ (only as a rough estimate). The range of potential difference given in Table 2 indicates a high probability of flashover either for first or for subsequent strokes.

The ground end currents for the ground wires are given in Table 3. Their magnitudes are quite comparable to that for isolated cylindrical down conductor. The highest amplification of the ground end current, which occurs for fast front currents, is about 1.9 times. These currents exhibit oscillation and have many similar characteristics as that of the potential. A good earth termination has been presumed in the simulation. It is possible that even in designs with higher terminating resistance, the soil ionisation would set in to reduce the effective value. Therefore, above deductions apply quite generally.

The induced tower base currents are presented in Table 4. Its basic features can be summarised as:

- $\quad$ The induced tower base currents for fast front strokes (which are typical of subsequent strokes) assume 30 $80 \%$ of the incident stroke current.

- Induction to the tower increases with proximity of the ground wire. This can be attributed to increase in level of coupling to the tower.

- $\quad$ As can be expected, the induced tower currents are in opposite direction to the ground wire currents.

Sample simulation results for configuration with $10 \mathrm{~m}$ long mast are given in Table $\mathbf{5}$ and it can be verified that they are quite similar to the previous case. Incidentally, as compared to the case of $5 \mathrm{~m}$ insulator, at many columns the peak voltage for this case can be seen to be lower for fast front currents and larger for slow front current waves. This feature can be basically attributed to the reaction field produced at the tower top. The incident electromagnetic field induces current in the tower, which produces a reaction field opposing the incident one. The initial unipolar current, as per the continuity equation, charges the tower and this charge assumes peak value at the tower top. In the due course, the time variation in the stroke current reduces and hence will be the level of induction to the tower. Then the induced charge in the tower becomes dominant and will cause current in opposite direction. The resulting oscillation would continue till the energy is dissipated [19].

This charge is found to aid the field at the tower top. Accordingly, a higher induced current would result in higher charge deposition and hence a higher resultant field. While for the slow front current waves, the level of induction is lower and therefore the role of tower in reducing the field would be mostly dependent on its relative position. This is the reason for the above deviation. As mentioned earlier, insulating cylinder of $10 \mathrm{~m}$ length is more difficult to realize. In view of these, for the remaining analysis, only $5 \mathrm{~m}$ long insulating cylinder is considered.

\subsubsection{Dependency on Radius of Ground Wire}

As mentioned earlier, the ground wire radius of $6 \mathrm{~mm}$ has been employed for the simulation. In order to ascertain the influence of the radius of ground wire on the results, simulations are carried out with ground wires of 1 and $2 \mathrm{~cm}$ radii. It would be impractical to consider wires of radius larger than the above.

Table 4. Peak Values of Induced Tower Base Currents in Ampere for Insulated Mast Design with One Ground Wire (Stroke Current Peak Amplitude Normalised to Unity \& Insulation Cylinder Length = $5 \mathrm{~m})$

(a) Grounding Position P1

\begin{tabular}{|c|c|c|c|c|}
\hline Support Tower Height (m) & \multirow{2}{*}{45} & \multirow{2}{*}{60} & \multirow{2}{*}{90} & \multirow{2}{*}{120} \\
\hline Rise Time $(\mu \mathrm{s})$ & & & & \\
\hline 0.26 & 0.34 & 0.42 & 0.51 & 0.57 \\
\hline 0.75 & 0.14 & 0.21 & 0.33 & 0.42 \\
\hline 1 & 0.08 & 0.14 & 0.25 & 0.35 \\
\hline 2.4 & 0.02 & 0.03 & 0.06 & 0.12 \\
\hline 4.87 & 0.01 & 0.02 & 0.02 & 0.04 \\
\hline
\end{tabular}

(b) Grounding position P2

\begin{tabular}{|c|c|c|c|c|}
\cline { 1 - 3 } Support Tower Height (m) & \multirow{2}{*}{$\mathbf{4 5}$} & $\mathbf{6 0}$ & $\mathbf{9 0}$ & \multirow{2}{*}{$\mathbf{1 2 0}$} \\
\cline { 1 - 4 } Rise Time $(\boldsymbol{\mu s})$ & & & & \\
\hline \hline 0.26 & 0.31 & 0.43 & 0.57 & 0.65 \\
\hline 0.75 & 0.12 & 0.23 & 0.40 & 0.54 \\
\hline 1 & 0.01 & 0.14 & 0.31 & 0.45 \\
\hline 2.4 & 0.01 & 0.01 & 0.03 & 0.04 \\
\hline 4.87 & & &
\end{tabular}

(c) Grounding Position P3

\begin{tabular}{|c|c|c|c|c|}
\hline Support Tower Height (m) & \multirow{2}{*}{45} & \multirow{2}{*}{60} & \multirow{2}{*}{90} & \multirow{2}{*}{120} \\
\hline Rise Time $(\mu s)$ & & & & \\
\hline 0.26 & 0.32 & 0.45 & 0.62 & 0.76 \\
\hline 0.75 & 0.14 & 0.21 & 0.40 & 0.55 \\
\hline 1 & 0.05 & 0.12 & 0.29 & 0.45 \\
\hline 2.4 & 0.02 & 0.02 & 0.05 & 0.09 \\
\hline 4.87 & 0.01 & 0.01 & 0.02 & 0.04 \\
\hline
\end{tabular}

Table 6 present the results for one ground wire scheme earthed at position $\mathrm{P} 1$, however with different wire radii. The length of insulation cylinder is $5 \mathrm{~m}$ and height of the tower is $120 \mathrm{~m}$. Similar to that reported in [3], there seems to be no appreciable dependency of the results on the radius of ground wire. It may be worth recalling here that the impulse corona has been neglected in the analysis and its effect can be expected to be almost identical in all the three cases. Therefore, at present, mechanical consideration may be given more prominence in selecting the ground wire radius.

The potential rise for the single ground wire scheme discussed above can be seen to be quite high. For its possible 
Table 5. Results for Insulation Cylinder Length $=10 \mathrm{~m}$ (Stroke Current Peak Amplitude Normalised to Unity \& One Ground Wire Earthed at P1)

(a) Potential rise Across the Insulating Cylinder

\begin{tabular}{|c|c|c|c|c|}
\hline \multirow{yynn}{*}{$\begin{array}{c}\text { Support Tower Height (m) } \\
\text { Rise Time }(\boldsymbol{\mu s})\end{array}$} & \multirow{4}{*}{$\mathbf{6 5}$} & $\mathbf{6 0}$ & $\mathbf{1 2 0}$ \\
\hline \hline 0.26 & 575 & 574 & 701 & 756 \\
\hline 0.75 & 370 & 454 & 653 & 712 \\
\hline 1 & 252 & 360 & 577 & 654 \\
\hline 2.4 & 83 & 126 & 216 & 317 \\
\hline 4.87 & 43 & 62 & 82 & 108 \\
\hline
\end{tabular}

\section{(b) Ground End Currents of Ground Wire}

\begin{tabular}{|c|c|c|c|c|}
\cline { 1 - 4 } Support Tower Height (m) & \multirow{2}{*}{$\mathbf{4 5}$} & $\mathbf{6 0}$ & $\mathbf{9 0}$ & $\mathbf{1 2 0}$ \\
\cline { 1 - 4 } Rise Time $(\boldsymbol{\mu s})$ & 1.91 & 1.93 & 1.92 & 1.93 \\
\hline \hline 0.26 & 1.65 & 1.80 & 1.90 & 1.92 \\
\hline 0.75 & 1.43 & 1.63 & 1.84 & 1.91 \\
\hline 1 & 1.04 & 1.09 & 1.25 & 1.45 \\
\hline 2.4 & 1.01 & 1.09 & 1.04 & 1.08 \\
\hline 4.87 & & &
\end{tabular}

(c) Induced Tower Base Current

\begin{tabular}{|c|c|c|c|c|}
\cline { 1 - 4 } Support Tower Height $(\mathbf{m})$ & \multirow{2}{*}{$\mathbf{4 5}$} & $\mathbf{6 0}$ & $\mathbf{9 0}$ & $\mathbf{1 2 0}$ \\
\cline { 1 - 4 } Rise Time $(\boldsymbol{\mu s})$ & 0.29 & 0.37 & 0.45 & 0.51 \\
\hline \hline 0.26 & 0.11 & 0.18 & 0.29 & 0.38 \\
\hline 0.75 & 0.07 & 0.12 & 0.23 & 0.31 \\
\hline 1 & 0.01 & 0.03 & 0.06 & 0.11 \\
\hline 2.4 & 0.01 & 0.01 & 0.03 & 0.04 \\
\hline 4.87 & &
\end{tabular}

reduction multiple ground wire scheme seems to be a possible solution. However, as discussed above, the mechanical stress on the insulator and space for the ground wire may put severe constraint on addition of ground wires. In the following, schemes with two and four ground wires will be dealt with. As the possible range of configuration is quite high, only demonstrative exercises will be carried out. It has been demonstrated in the earlier work [3] that spacing between the ground wires is not very critical and hence no study in this direction will be made. For a given grounding location, the ground wires are displaced horizontally with an angle of $45^{\circ}$.

\subsection{Insulating Mast with Two Ground Wires}

It has been discussed previously that the ground wire scheme is slightly modified and represented for the numerical field computation. The first segment of the ground wire connected to the mast is made parallel to the ground. In the numerical representation of the two and four ground wire schemes, this horizontal member is left as it is and the multiple ground wires are made to start from the other end of this member.

Table 6. Comparison of Peak Values of the Parameters for One Ground Wire Scheme with Different Wire Radii (Stroke Current Peak Amplitude Normalised to Unity. $V_{\text {Top }}$ is the Potential Across Insulation, $\mathbf{I}_{\mathrm{GW}}$ is the Ground End Currents of Ground Wire and $I_{T B}$ is the Induced Tower Base Current)

(a) Wire Radius $=6 \mathrm{~mm}$

\begin{tabular}{|c|c|c|c|}
\hline Rise Time ( $\boldsymbol{\mu s )}$ & $\mathbf{V}_{\text {Top }}$ & $\mathbf{I}_{\mathbf{G W}}$ & $\mathbf{I}_{\mathbf{T B}}$ \\
\hline \hline 0.26 & 734 & 1.93 & 0.57 \\
\hline 0.75 & 685 & 1.92 & 0.42 \\
\hline 1 & 649 & 1.91 & 0.35 \\
\hline 2.4 & 331 & 1.45 & 0.12 \\
\hline 4.87 & 134 & 1.09 & 0.04 \\
\hline
\end{tabular}

(b) Wire Radius $=1 \mathrm{~cm}$

\begin{tabular}{|c|c|c|c|}
\hline Rise Time ( $\boldsymbol{\mu s )}$ & $\mathbf{V}_{\text {Top }}$ & $\mathbf{I}_{\mathbf{G W}}$ & $\mathbf{I}_{\mathbf{T B}}$ \\
\hline \hline 0.26 & 733 & 1.92 & 0.57 \\
\hline 0.75 & 685 & 1.91 & 0.42 \\
\hline 1 & 649 & 1.90 & 0.35 \\
\hline 2.4 & 316 & 1.45 & 0.12 \\
\hline 4.87 & 107 & 1.08 & 0.04 \\
\hline
\end{tabular}

(c) Wire Radius $=2 \mathrm{~cm}$

\begin{tabular}{|c|c|c|c|}
\hline Rise Time ( $\boldsymbol{\mu s )}$ & $\mathbf{V}_{\text {Top }}$ & $\mathbf{I}_{\mathbf{G W}}$ & $\mathbf{I}_{\mathbf{T B}}$ \\
\hline \hline 0.26 & 707 & 1.92 & 0.56 \\
\hline 0.75 & 659 & 1.91 & 0.41 \\
\hline 1 & 623 & 1.89 & 0.35 \\
\hline 2.4 & 302 & 1.44 & 0.12 \\
\hline 4.87 & 103 & 1.08 & 0.04 \\
\hline
\end{tabular}

Table 7 presents results for insulating mast design with two ground wires, all the ground wires earthed at P1. With addition of second catenary/ground wire, the potential rise has been considerably reduced. As before, when the two-way travel time on the ground wires is larger than the time to peak of the stroke current, the rise in potential is considerable. Also, for such situations, the potential rise is higher for taller support towers.

The ground end currents of the ground wires are found to be similar in magnitude and hence are not presented. In contrast to the potential, there is no appreciable reduction in the induced tower base currents. For some cases they were lower by about $\cong 15 \%$. 
Table 7. Potential Rise Across Insulation Support in Volts for Design with Two Ground Wires Earthed at P1 (Insulation Cylinder Length $=5 \mathrm{~m}$; Stroke Current Peak Amplitude Normalised to Unity)

\begin{tabular}{|c|c|c|c|c|}
\hline \multirow{2}{*}{ Rise Time ( $\boldsymbol{\mu s})$} & \multicolumn{4}{|c|}{ Tower Height } \\
\cline { 2 - 5 } & $\mathbf{4 5} \mathbf{~ m}$ & $\mathbf{6 0} \mathbf{~ m}$ & $\mathbf{9 0} \mathbf{~ m}$ & $\mathbf{1 2 0} \mathbf{~ m}$ \\
\hline \hline 0.26 & 200 & 192 & 212 & 213 \\
\hline 0.75 & 104 & 130 & 171 & 199 \\
\hline 1.0 & 72 & 98 & 143 & 175 \\
\hline 2.4 & 56 & 53 & 59 & 78 \\
\hline
\end{tabular}

\subsection{Insulating Mast with Four Ground Wires}

With two ground wires the potential rise is substantially reduced, however, it seems to be still higher than the rise in potential for the case of support tower working as the down conductor. For a further reduction in the potential rise, fourground wire configuration is studied. All the four ground wires have been earthed on the same side of the tower, owing to the constraint for space in practical scenario. Two are grounded at $\mathrm{P} 1$ and other two at $\mathrm{P} 2$.

Table 8. Potential Rise Across Insulation Support in Volts for Design with Four Ground Wires (Insulation Cylinder Length $=5 \mathrm{~m}$; Stroke Current Peak Amplitude Normalised to Unity)

\begin{tabular}{|c|c|c|c|c|}
\hline \multirow{2}{*}{ Rise Time ( $\boldsymbol{\mu s )}$} & \multicolumn{4}{|c|}{ Tower Height } \\
\cline { 2 - 5 } & $\mathbf{4 5} \mathbf{~ m}$ & $\mathbf{6 0 ~} \mathbf{~}$ & $\mathbf{9 0} \mathbf{~ m}$ & $\mathbf{1 2 0} \mathbf{~ m}$ \\
\hline \hline 0.26 & 354 & 374 & 394 & 402 \\
\hline 0.75 & 194 & 273 & 341 & 363 \\
\hline 1.0 & 120 & 202 & 302 & 338 \\
\hline 2.4 & 35 & 51 & 102 & 159 \\
\hline
\end{tabular}

Table 8 presents the simulation results. It can be seen that there is a considerable reduction in rise in potential and it is almost in the same range as the rise in potential for the case of support tower working as down conductor. As before, the potential rise increases with height of the support tower. There is no significant difference in the induced tower base currents.

Before any further scrutinisation of this scheme, a discussion on the model employed for the excitation will be made. This is believed to provide further support to the modelling and hence the simulation results.

\section{A DISCUSSION ON MODEL EMPLOYED FOR THE EXCITATION}

Some justifications for the use of current source model for the excitation have been provided in section 2.2.1. A further discussion will be made here. After investigation on the measured stroke currents on tall objects, it is concluded in [2] that the peak current measured at the top of the struck object is not significantly affected. This conclusion is applied to objects of even 40 and $70 \mathrm{~m}$ height. In other words, it appears to be independent of two-way travel time on the struck object. Under the regime of lumped source modelling with voltage source excitation, the above condition could be met, irrespective of the source resistance (varied between $600 \Omega$ to $5 \mathrm{k} \Omega$ ), by a two step process-first calculate the voltage to produce desired current at the injection point and then use it for the required calculations. Such an approach can be verified to yield results, which are very close to the results provided by the current source model.

On the other hand, if a voltage source model along with a resistance is considered, the waveshape of the source current cannot be maintained. It will possess superimposed oscillations. Therefore it cannot be considered for the evaluation.

Only for the cases in which the two way travel time along the ground wire is greater than the time to peak of the current, actual peak of the current could be correctly identified. Simulations for such cases have shown that when the source resistance is varied from $600 \Omega$ to $5 \mathrm{k} \Omega$, the differences in peak potential rise and ground wire currents are well within $15 \%$. In these simulations, the strength of the voltage source was adjusted so as to get same peak value for the current. As can be expected, with lower source resistance, the oscillatory nature of the potential and ground wire currents decreased significantly. However for scheme with multiple ground wires, owing to the smaller "surgeimpedance" of the system, the above differences diminished with increase in number of ground wires. With four ground wires, both current source model and the voltage source model with even $600 \Omega$ source resistance are found to yield almost the same results. These additional details seem to provide further support to the source model employed in this work.

\section{FURTHER DISCUSSION ON INSULATED MAST SCHEME}

Some pertinent discussion on this scheme has been presented in [3]. The success of this scheme is solely dependent on the withstand capabilities of the two critical gaps. As mentioned earlier, the first gap involves catenary/ground wire and the tower top. The length of this air gap is dependent on the earthing location of ground wire. For the nominal grounding locations, it will be close to the length of the insulating support. The second gap is along the insulating cylinder. It is well known in insulation engineering that once a surface is introduced, the net electrical strength can go below that of the original air gap itself. In other words, the bulk breakdown strength of the insulating support will not be of any concern at all. Instead, it is the surface breakdown strength along the insulator surface that needs to be considered. This surface strength, similar to the electrical power transmission line pollution flashover problems, could be affected by the natural/artificial pollution [3]. Data on oscillatory impulse flashover strength (whether dry or wet) of 10-15 m long smooth surfaces (i.e. without any sheds) seems to be rather scarce and it is more so under polluted conditions. Depending on the type of insulator material, it may also become necessary to consider the effect of space charge accumulation around the surface. 
Much more work is necessary to ascertain the actual withstand capabilities of these two gaps.

In summary, the prevailing significant induction to the tower and the limited breakdown strength of the two critical gaps, seem to disfavour the original philosophy of the insulated mast scheme.

\section{SUMMARY}

The insulated mast scheme for the lightning protection system has been employed in practice. Many advantages over conventional protection schemes are sometimes envisaged. However, the technical literature on the analysis of such schemes is rather scarce. As a first step to address this problem, the present work was taken up and the potential rise at the top and ground end currents in the insulating mast scheme with single tower have been investigated for a wide range of tower heights and pertinent values of other parameters. The results for $120 \mathrm{~m}$ tall support tower are in line with the earlier work.

The important findings of the work are:

- There is a considerable potential rise at the top and it gets enhanced for strokes with time to crest lower than two way travel time along the ground wire(s). Also it is higher for taller support towers and for ground wire earthed at far distances.

- $\quad$ The magnitude of the potential rise, (as compared to the case of tower itself acting as a down conductor) is quite high for one ground wire scheme. However with multiple ground wires, the potential rise can be reduced. With four-ground wire scheme, it can be made comparable to that on the support tower serving as the down conductor. Mechanical considerations constraint the number of ground wires.

- The potential rise at the top and the ground end currents of ground wires exhibits sustained oscillations, whose frequency is determined by the length of the ground wires. This may be partly attributed to the consequence of a current source representation for the excitation.

- Even when the support tower is electrically isolated, it possesses significant induced currents. For fast front stroke currents, which is typical of subsequent return strokes, the induced tower base currents can reach, depending on the height of the tower, $30-80 \%$ of the incident stroke current.

The level of induction into the support tower and uncertainty in ensuring no flashover to the same is not in favour of this scheme.

\section{REFERENCES}

[1] Jyothirmayi R. Investigations on the lightning surge response of isolated down conductor. M. Sc. (Eng.) Thesis, Dept. of Electrical Engineering, Indian Institute of Science, India 2006.

[2] Rakov VA. Transient response of a tall object to lightning. IEEE Intern Electric Electron Eng Transac Electromagn Compatibil 2001; 43(4): 654-61.

[3] Kumar U, Nayak PK. Investigations on the post-stroke voltages and currents in lightning protection schemes involving single tower. IEEE Trans Intern Electric Electron Eng Transac Electromagn Compatibil 2005; 47(3): 543-51.

[4] Grcev AL, Rachidi F. On tower impedances for transient analysis. IEEE Trans Power Del 2004; 19(3): 1238-44.

[5] Chisholm WA, Chow YL, Srivastava KD. Lightning surge response of transmission towers. IEEE Trans Power Appl Syst 1983; 102 (9): 3232-42.

[6] Kawai M. Studies of the surge response of a transmission line tower. IEEE Trans Power Appl Syst 1964; 83: 30-4.

[7] Anderson JG, Hagenguth JH. Magnetic fields around a transmission line tower. IEEE Trans Power Appl Syst 1959; 78: 1391-8.

[8] Wagner CF, Hileman AR. A new approach to the calculation of the lightning performance of transmission lines III-A simplified method: stroke to tower. IEEE Trans Power Appl Syst 1960; 79: 589-603.

[9] Sargent MA, Darveniza M. Tower surge impedance. IEEE Trans Power Appl Syst 1969; 88(5): 680-7.

[10] Motoyama H, Matsubara H. Analytical and experimental study on surge response of transmission line tower. IEEE Trans Power Del 200; 15(2): 812-9.

[11] Rakov VA, Uman MA. Review and evaluation of lightning return stroke models including some aspects of their application. IEEE Intern Electric Electron Eng Transac Electromagn Compatibil 1998; 40(4): 403-26.

[12] Ishii M, Baba Y. Numerical electromagnetic field analysis of tower surge response. IEEE Trans Power Del 1997; 12(1): 916-21.

[13] Baba Y, Ishii M. Numerical electromagnetic field analysis on measuring methods of tower surge response. IEEE Trans Power Del 1999; 14(2): 630-5.

[14] Kordi B, Moini R, Janischewskyj W, Hussein AM, Shostak V, Rakov VA. Application of the antenna theory model to a tall tower struck by lightning. J Geophys Res 2003; 108(D17): 4542.

[15] Burke GJ, Poggio AJ. Numerical Electromagnetic Code (NEC)method of moments part I \& III. Technical document 116, San Diego: Naval Ocean Systems Center 1980.

[16] Miller EK, Burke GJ. Low-Frequency computational electromagnetics for antenna analysis. Proc IEEE 1992; 80(1): 2443.

[17] Ishii M, Baba Y. Advanced computational methods in lightning performance-the numerical electromagnetic code (NEC-2). Proc. IEEE PES Winter Meeting; Singapore 2000; p. 2419-24.

[18] Kumar U, Hegde V, Shivanand V. IEC 61024-1. Protection of Structures Against Lightning-Part 1: General Principles 1990-03. Preliminary studies on the characteristics of the induced currents in simple down conductors due to a nearby lightning strike. IEEE Intern Electric Electron Eng Transac Electromagn Compatibil 2006; 48(4): 805-6. 